Erschienen in: Engelberg, Stefan/Holler, Anke/Proost, Kristel (Hrsg.):

Sprachliches Wissen zwischen Lexikon und Grammatik. - Berlin, Boston: de

Gruyter, 2011. S. 317-343. (Institut für Deutsche Sprache. Jahrbuch 2010), https://doi.org/10.1515/9783110262339.317

Claudia Maienborn

\title{
Strukturausbau am Rande der Wörter: Adverbiale Modifikatoren beim Zustandspassiv ${ }^{1}$
}

\begin{abstract}
Der Beitrag bietet einen Analysevorschlag für ereignisbezogene adverbiale Modifikatoren beim Zustandspassiv, der ihr Auftreten in verbaler Umgebung mit der adjektivischen Natur des Zustandspassivs in Einklang bringt. Grundlage hierfür ist eine empirisch breit abgesicherte Argumentation für das Vorliegen einer besonderen strukturellen Nähe zwischen Modifikator und Partizip, die gleichsam eine kompakte Einheit in der Übergangszone zwischen Wort und Phrase bilden. Diese Sicht macht den Weg frei für eine strikt kompositionale Semantik des Zustandspassivs samt adverbialen Modifikatoren.
\end{abstract}

\section{Das Problem}

Mit dem Tagungsthema ,Sprachliches Wissen zwischen Lexikon und Gram-

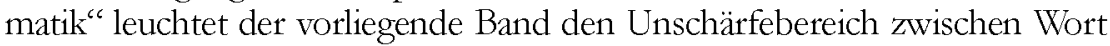
und Phrase aus. Dieser Beitrag bietet eine Studie zu einem Ausdruckstyp, der bislang noch nicht in diesem Feld verortet wurde, gleichwohl aber - so meine Argumentation - hier anzusiedeln ist. Es handelt sich um die Kombination des Zustandspassivs mit bestimmten adverbialen Modifikatoren vom Typ (1a). Diese werfen Probleme für aktuelle adjektivische Zustandspassivanalysen auf. Eine Würdigung des besonderen strukturellen und interpretativen Status dieses Ausdruckstyps in Abgrenzung von klaren verbalen Konstruktionen wie dem Vorgangspassiv in (1b) und in Relation zu Wortbildungsmustern wie in (1c) eröffnet einen neuen Erklärungsansatz für die problematischen Zustandspassivdaten als Strukturausbau am Rande der Wörter und liefert zugleich eine verbreiterte empirische Basis zur Bestimmung der Strukturbildungsbedingungen und -optionen in der Übergangszone zwischen Lexikon und Grammatik.

(1) a. Das Manuskript ist von Chomsky zitiert.

b. Das Manuskript ist von Chomsky zitiert worden.

c. Das Manuskript ist Chomsky-zitiert.

Für Brigitte! Dieser Beitrag ist im Rahmen des DFG-Projekts A1 „Kombinatorische Bedeutungsvariation an der Semantik/Pragmatik-Schnittstelle im Tübinger SFB 833 „Bedeutungskonstitution" entstanden. Ich danke meinen Projektmitarbeiterinnen, Helga Gese und Britta Stolterfoht, sowie Stefan Engelberg, Werner Frey, Torgrim Solstad und dem Auditorium der IDS-Jahrestagung 2010 für anregende Diskussionen und hilfreiche Kommentare. 
Die heutige Sicht auf das so genannte "Zustandspassiv" ist weitgehend durch die Annahme geprägt, dass es sich hierbei um die Verbindung der Kopula sein mit einem adjektivierten Partizip II (und somit um kein Genus verbi) handle (siehe insbesondere Kratzer 1994, 2000; Rapp 1996, 1997; Maienborn 2007, 2009). Problematisch für diese Adjektiv-Auffassung ist das Auftreten bestimmter adverbialer Modifikatoren, die typischerweise in verbalen Kontexten, nicht aber bei Adjektiven auftreten. So finden sich beim Zustandspassiv insbesondere Agens- (2), Instrumental- (3) und Lokalangaben (4). ${ }^{2}$ Reine Adjektiv-Konstruktionen lassen derartige Erweiterungen typischerweise nicht zu.

(2) a. Die Zeichnung ist von einem Kind angefertigt. Agensangabe b. * Die Zeichnung ist von einem Kind schön.

(3) a. Der Brief war mit roter Tinte geschrieben. Instrumentalangabe b. * Der Brief war mit roter Tinte leserlich.

a. Die Birnen waren in Rotwein gedünstet.

b. * Die Birnen waren in Rotwein weich.

Lokalangabe

Offensichtlich beziehen sich die Modifikatoren der a-Sätze in (2)-(4) auf das vom Basisverb bezeichnete Ereignis. Dieses scheint als Andockstelle für den Modifikator noch zugänglich zu sein. Das Partizip schüttelt also seine verbale Herkunft offenbar nicht vollständig ab.

Allerdings ist in der Literatur auch immer wieder darauf hingewiesen worden (z.B. in Vaagland 1983; Litvinov/Nejalkov 1988; Rapp 1996), dass derartige Kombinationen keinesfalls systematisch verfügbar seien, sondern vielmehr massiven Beschränkungen unterliegen, wie sie im Vergleich dazu etwa beim Vorgangspassiv nicht auftreten; siehe die Beispiele in (5).

a. * Die Tür ist von ihm geöffnet.

(Vaagland 1983, S. 194)

b. * Ihre Haare sind mit einem goldenen Kamm gekämmt.

c. * Sie ist im Nachbarwald umgebracht.

(Rapp 1996, S. 257)

(Litvinov/Nedjalkov 1988, S. 139)

Dies macht eine Erklärung der zulässigen Kombinationen in (1a)-(4a) umso schwieriger. Im Folgenden soll ein Vorschlag im Rahmen der Adjektivierungsanalyse des Zustandspassivs entwickelt werden, der die Zulässigkeit der Konstellation vom Typ (1a) auf die mit der Adjektivierung einhergehenden besonderen Interpretationsbedingungen des Zustandspassivs zurückführt und ihren Platz bestimmt zwischen der unzweifelhaft phrasalen Verbindung von Vorgangspassiv und adverbialem Modifikator in (1b) und dem Wortbildungsmuster in (1c).

Siehe auch die umfangreiche Aufstellung in Litvinov/Nedjalkov (1988, Kap. 5). 
Der folgende Abschnitt 2 bietet eine kurze Rekapitulation der Gründe, die für eine Adjektivierungsanalyse des Zustandspassivs sprechen. Abschnitt 3 geht auf bisherige Vorschläge, adverbiale Modifikatoren in diesem Rahmen zu erfassen, ein. In Abschnitt 4 lege ich meine Annahmen zur Semantik des Zustandspassivs dar. Dies bildet die Grundlage für die Diskussion der strukturellen Integration adverbialer Modifikatoren beim Zustandspassiv und den daraus abzuleitenden Schlussfolgerungen in den Abschnitten 5 und 6.

\section{Zur Adjektivierungsanalyse des Zustandspassivs}

In den zurückliegenden Jahren hat eine intensive Auseinandersetzung mit der Frage nach der grammatischen Einordnung des so genannten Zustandspassivs stattgefunden. Überblicke und Bewertungen der in Betracht gezogenen Optionen finden sich in Rapp (1996) und Maienborn (2007). Ich will die Argumente für und wider verschiedene Analysen hier nicht wiederholen, sondern lediglich mithilfe einiger ausgewählter Diagnostiken die heute in der Forschungsliteratur übliche, wenn auch in den meisten Grammatiken noch nicht reflektierte Sicht ${ }^{3}$ auf das Zustandspassiv als Kombination aus der Kopula sein mit einem adjektivierten Partizip begründen (siehe z.B. auch Wunderlich 1997; von Stechow 1998; Schlücker 2005).

Ein klares Indiz für die Kategorisierung des Partizips als Adjektiv ist die Möglichkeit der un-Affigierung (siehe Lenz 1993). Im Deutschen ist das Negationsaffix tm- (von der beschränkten Präfigierung von Nomina abgesehen) nur mit Adjektiven, nicht aber mit Verben kombinierbar. Das heißt, dass die Partizipien in (6) als Basen für die $u n$-Affigierung bereits adjektiviert sein müssen. ${ }^{4}$

(6) a. Oft waren diese Regeln ungeschrieben, aber sie waren trotzdem jedem klar.

(TIGER s11406)

b. Mein Haar ist ungekämmt und mein Hemdkragen ungebügelt.

(COSMAS M89/909.31688)

Für die adjektivische Natur des Partizips beim Zustandspassiv spricht auch die Möglichkeit zur Graduierung wie in (7).

Siehe aber die Abwägung der Argumente in Zifonun et al. (1997, S. 1822 f.). Letztlich ist das Auftreten adverbialer Modifikatoren eines der Hauptargumente für Zifonun et al., trotz aller Adjektiv-Evidenz an der traditionellen Analyse des Zustandspassivs als Verbform festzuhalten.

$4 \quad$ Angaben zu elektronischen Korpusbelegen beziehen sich auf das morphosyntaktisch annotierte TIGER-Korpus (www.ims.uni-stuttgart.de/projekte/TIGER) und die COSMAS-Korpora des IDS Mannheim (www.ids-mannheim.de/cosmas2, beide Stand: 06.12.2010). 
(7) a. ... Autor, der viel berühmter und vor allem weitaus gelesener war als Goethe.

b. Am bedrohtesten sind die Landtage.

(Die Zeit, 29.07.1999)

(COSMAS V98/MAI.21411)

Zwar ist Graduierung beim Zustandspassiv nur begrenzt möglich - dies ist einer der Gründe, warum Zifonun et al. (1997, S. 1822 f.) letztlich doch an der Auffassung, es handle sich um Verbformen, festhalten -, wegen der verbalen Herkunft der betreffenden Partizipien und der damit einhergehenden Beschränkungen und Besonderheiten gegenüber genuinen Adjektiven ist dies aber auch gar nicht anders zu erwarten, zumal auch unzweifelhafte Adjektive Graduierung nicht immer zulassen (siehe Lübbe 2004). Schon die Existenz von offenbar regulär gebildeten Fällen vom Typ (7) muss als ein gewichtiges Argument für eine dem Zustandspassiv zugrunde liegende Adjektivierung des Partizips gewertet werden.

Ein weiteres Adjektivierungsindiz liefern Koordinationsdaten wie in (8). Koordiniert werden kann nur Gleichartiges (siehe Lang 1984). In Verbindung mit einem genuinen Adjektiv kann die sein-Form nicht zugleich Kopula und Auxiliar sein, sondern ist auf die Kopula-Variante festgelegt.

a. Seine Schuhe waren sauber und poliert.

b. Entlassungen seien weder geplant noch nötig.

(TIGER s3166)

Und schließlich finden sich beim Zustandspassiv typisch adjektivische Wortbildungen vom Typ in (9), die im verbalen Bereich wiederum ausgeschlossen sind. ${ }^{5}$

(9) a. Dieses Geschäft ist videoüberwacht.

b. Alle Mitglieder des rot-roten Senats sind stasi-überprüft.

c. Leider waren unsere Gäste noch etwas kaufblockiert.

(Schwäbisches Tagblatt 15.10.2009)

Die Produktivität dieses Wortbildungsmusters zeigt sich auch an okkasionellen Bildungen wie Chomsky-zitiert sein in (1c) oder schwedentrainiert sein in (10). Ich werde darauf im Zusammenhang mit der Interpretation des Zustandspassivs zurückkommen.

(10) Ich hatte Sorge, wie der Japaner das Oktoberfest finden würde, aber es stellte sich heraus, dass er schwedentrainiert war.

(Hörbeleg 11/2007)

An dieser Stelle sei darauf hingewiesen, dass der attributive Gebrauch von Partizipien kein verlässlicher Indikator für ihren adjektivischen Status ist (siehe hierzu insbesondere Wunderlich 1997). Die Aussagen des vorliegenden Artikels betreffen allein den prädikativen Gebrauch des Partizips II, d.h. das Zustandspassiv. 
Die Liste der Adjektivierungsindizien ließe sich fortsetzen und auch psycholinguistisch untermauern. So zeigen Stolterfoht et al. (2010) im Rahmen eines Lesezeitexperiments, dass beim Partizip in der Zustandspassivkonstellation eine zeitliche Verzögerung gegenüber der Vorgangspassivkonstellation auftritt. Diesen Verarbeitungsmehraufwand werten sie als Evidenz für die mit dem Zustandspassiv verbundene zusätzliche Adjektivierung des verbalen Partizips.

In der Summe stützen die zusammengetragenen Diagnostiken zur kategorialen Bestimmung des Zustandspassivs übereinstimmend die Adjektivierungshypothese, derzufolge sich hinter dem Zustandspassiv die Kombination der Kopula sein mit einem adjektivierten Partizip verbirgt. Vor diesem Hintergrund ist die Redeweise vom ,Zustandspassiv" unglücklich gewählt; es handelt sich gerade nicht um eine passive Verbform als sozusagen „kleine Schwester ${ }^{c c}$ des Vorgangspassivs. ${ }^{6}$ Da dieser Terminus zur Bezeichnung des in Rede stehenden Phänomens sich inzwischen aber in der deutschsprachigen Literatur etabliert hat, werde ich ihn hier auch weiterhin verwenden.

\section{Lösungsvorschläge für das Auftreten adverbialer Modifikatoren}

Wenn nun aber die hier diskutierten , sein plus Partizip"-Kombinationen gar keine analytischen Verbformen sind, wie lässt sich dann das Auftreten typischer adverbialer Modifikatoren wie die Agens-, Instrumental- oder Lokalangaben in (2)-(4) erklären? ${ }^{7}$ Hierzu bietet Kratzer $(1994,2000)$ einen interessanten Vorschlag an. ${ }^{8}$ Danach gibt es zwei Möglichkeiten, das verbale Partizip in ein Adjektiv zu konvertieren (in (11) umgesetzt über die Annahme eines adjektivischen Nullaffixes). Die Adjektivierung kann entweder auf lexikalischer Ebene stattfinden oder aber auf phrasaler Ebene. Gegenstand der Adjektivierung ist im letzteren Fall nicht allein das Verb, sondern eine komplette Verbalphrase inklusive aller innerhalb der VP auftretenden Modifikatoren; vgl. die (vereinfachte) Darstellung in (11):

Siehe hierzu auch Gese et al. (2009, i.Dr.), die u.a. anhand der oben aufgeführten Diagnostiken zeigen, dass auch unakkusative Verben diesen Konstruktionstyp eingehen. Ein Satz wie (i) hat demnach neben der Perfekt-Lesart, bei der das Perfektauxiliar sein mit einem verbalen Partizip auftritt, auch eine „Zustandspassiv" ${ }^{c-L e s a r t ~ i n ~ G e s t a l t ~ d e r ~ K o m b i n a t i o n ~ a u s ~ K o p u l a ~}$ sein plus adjektiviertem Partizip. Zu den damit einhergehenden semantischen Unterschieden siehe Maienborn (2009).

(i) Meine Nachbarin ist verreist.

7 Zum Ausschluss von ereignisbezogenen Modifikatoren in Kopula-Konstruktionen siehe Maienborn (2003a, 2005).

8 Kratzers Vorschlag zur phrasalen Adjektivierung wurde verschiedentlich aufgegriffen und weiterentwickelt (siche z.B. von Stechow 1998; Anagnostopoulou 2003; Embick 2004; Alexiadou/Anagnostopoulou 2007). 
(11) a. Lexikalische Adjektivierung

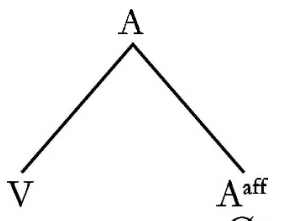

$\varnothing$

b. Phrasale Adjektivierung

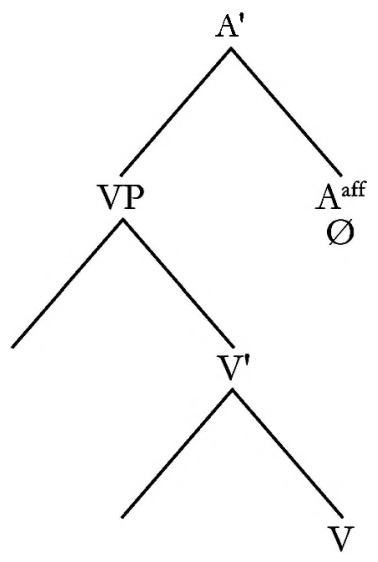

Das Auftreten adverbialer Modifikatoren beim Zustandspassiv kann damit in Kratzers Modell über die phrasale Adjektivierung erfasst werden. ${ }^{9}$ Allerdings wäre unter diesen Vorzeichen auch zu erwarten, dass via phrasale Adjektivierung beliebige innerhalb einer VP auftretende Modifikatoren auch beim Zustandspassiv grundsätzlich zulässig sein sollten. Dies trifft, wie die Beispiele in (5) zeigen, nicht zu.

Um diesem Problem der Übergenerierung zu begegnen, formuliert Rapp (1996, S. 257) die Zusatzbedingung, dass bei der phrasalen Adjektivierung nur solche adverbialen Modifikatoren zugelassen sind, die neben dem vom Basisverb bezeichneten Ereignis auch dessen Resultatszustand charakterisieren. So lässt sich beispielsweise beim Schreiben eines Briefes am Ergebnis in der Regel nicht mehr erkennen, ob der Brief langsam oder schnell geschrieben wurde, wohl aber, ob dabei rote Tinte zum Einsatz kam; vgl. (12a) vs. (12b).

Der Vorschlag erfasst zugleich die Inkompatibilität zwischen ereignisbezogenen Modifikatoren und der auf lexikalische Adjektivierung beschränkten un-Affigierung:

(i) * Das Hemd war von der Mutter ungebügelt.

(ii) $*$ Die Truhe war mit einem Brecheisen ungeöffnet.

Siehe hierzu aber die Gegenevidenz in Schlücker (2005). 
(12) a. * Der Brief war langsam geschrieben.

b. Der Brief war mit roter Tinte geschrieben.

Rapps Zusatzbedingung liefert einen ersten Hinweis auf die relevanten Lizensierungsbedingungen für adverbiale Modifikatoren beim Zustandspassiv, der im Weiteren inhaltlich und strukturell weiter präzisiert werden soll. Eine Übertragung auf unser Eingangsbeispiel (1a) (Das Manuskript ist von Chomsky qitiert.) macht deutlich, dass diese Bedingungen weiter geschärft werden müssen. Im Falle des Manuskript-Zitierens etwa ist nicht ohne weiteres klar, worin ein Resultatszustand des Zitiert-Seins genau besteht und inwiefern ein solcher Resultatszustand, in dem sich das Manuskript befindet, durch die zusätzliche Agensangabe von Chomsky näher charakterisiert würde. An dieser Stelle bedarf es eines genaueren Blicks auf die Interpretation des Zustandspassivs.

\section{Interpretation des Zustandspassivs}

Der in Maienborn (2007, 2009) entwickelte Vorschlag zur Semantik des Zustandspassivs setzt bei dessen grammatischer Einordnung an und leitet daraus die Grundzüge einer semantischen Analyse ab. Wenn das Zustandspassiv syntaktisch nichts anderes ist, als der Spezialfall einer Kopula-Konstruktion mit adjektivischem Prädikativ, so sollte dies auch für seine Semantik gelten. Wie bei allen anderen Kopula-Konstruktionen auch besteht sein semantischer Beitrag damit in der Zuschreibung einer Eigenschaft an den Subjektreferenten (siehe Maienborn 2003a, 2005). Die zweifellos besonderen Interpretationsbedingungen des Zustandspassivs wären demnach zurückzuführen auf die semantische Umsetzung des mit der Adjektivierung des verbalen Partizips einhergehenden grammatischen Kategorienwechsels und dessen pragmatischer Deutung.

Meine These zum Zustandspassiv ist, dass die Adjektivierung des Partizips zur Bildung einer pragmatisch salienten Ad hoc-Eigenschaft dient, welche aus dem vom Basisverb bezeichneten Ereignis resultiert. Während also Standard-Kopulasätze mit adjektivischem Prädikativ dem Subjektreferenten eine lexikalisch kodierte Eigenschaft zuschreiben, die einen fest vorgegebenen Platz im Eigenschaftsraum des Subjektreferenten einnimmt, ist das Zustandspassiv ein grammatisches Ausdrucksmittel, um (mehr oder weniger) ad hoc potenziell neue, ereignisbasierte Eigenschaften zu bilden, deren genaue Kontur und Platzierung im Eigenschaftsraum durch Kontext und Weltwissen mitbestimmt wird.

Was ist gemeint mit ,ereignisbasierten Ad hoc-Eigenschaften"? Betrachten wir dazu zunächst Beispiel (13):

Das Manuskript ist eingereicht. 
Satz (13) besagt mehr, als dass es ein in der Vergangenheit liegendes Ereignis des Manuskript-Einreichens gibt. Aufgrund unseres reichen Hintergrundwissens über Entstehungsstadien und Bewertungen von Publikationen im Wissenschaftsbetrieb können wir (13) unter Heranziehung potenzieller Alternativen als Aussage nicht nur über den Zustand, sondern auch die Güte des Manuskripts verstehen. Für eine Projektbegutachtung beispielsweise macht es einen qualitativen Unterschied, ob ein Manuskript angefangen, eingereicht, begutachtet, akzeptiert, zurückgezogen oder abgelehnt ist. Ein akzeptiertes Manuskript ist allemal besser als ein bloß eingereichtes. Ereignisbezogene Modifikatoren - dies kann an dieser Stelle schon vorweggenommen werden - erlauben eine weitergehende Spezifizierung und Differenzierung solcher Ad hoc-Eigenschaften.

(14) a. Das Manuskript ist bei Nature eingereicht.

b. Das Manuskript ist von Chomsky zitiert.

c. Das Manuskript ist in einer Nacht geschrieben.

Die Modifikatoren in (14) aktivieren jeweils Portionen von Hintergrundwissen, die Schlussfolgerungen auf die Art von Manuskript, mit der wir es zu tun haben, zulassen. In (14a) können wir beispielsweise aus der Reputation der Zeitschrift Nature schließen, dass das Manuskript von hoher Qualität sein muss - zumindest nach Ansicht des Autors, andernfalls hätte er nicht die Einreichung erwogen. Ähnliches gilt für (14b): Von Chomsky zitiert zu werden, gilt innerhalb weiter Teile der linguistischen Gemeinschaft als Ritterschlag. Und in (14c) lässt sich für ein Manuskript, das in einer Nacht geschrieben wurde, beispielsweise schlussfolgern, dass es aus einem Guss ist - oder aber ausgesprochen schlampig geschrieben.

Die Diskussion der Beispiele in (14) zeigt, dass die jeweiligen Schlussfolgerungen auf die dem Manuskript letztlich zuzuschreibende Eigenschaft je nach Kontext, Weltwissen und subjektiven Einstellungen erheblich variieren können. Eine vollständige Rekonstruktion dieser Zusammenhänge gehört sicher nicht zu den Aufgaben der Linguistik. Der mir wichtige Punkt hierbei ist, dass die Semantik des Zustandspassivs so angelegt ist, dass wir eine solche Ad hoc-Eigenschaft erschließen mïssen: Dem Subjektreferenten wird eine Eigenschaft zugewiesen, die in einer über Weltwissen festgelegten Beziehung zu dem vom Verb bezeichneten Ereignis steht. Diese Eigenschaft erschöpft sich nicht darin, am Nachzustand oder Resultatszustand des betreffenden Ereignisses beteiligt zu sein. Die Gegenüberstellung von Zustandspassiv und Perfekt des Vorgangspassivs in (15) kann dies verdeutlichen.

a. Das Manuskript ist von Chomsky zitiert.

b. Das Manuskript ist von Chomsky zitiert worden.

Mit (15a) wird dem Subjektreferenten eine semantisch unterbestimmte Ad hoc-Eigenschaft zugeschrieben, die aus einem (in der Regel) vergangenen 
Ereignis resultiert. Das Manuskript wird als zur Klasse der von Chomsky zitierten Schriften gehörig kategorisiert, wobei der Hörer gefordert ist, die charakteristischen Merkmale dieser Klasse zu bestimmen. Mit (15b) wird ausgedrückt, dass wir uns im Nachzustand eines Zitierensereignisses befinden, an dem Chomsky als Agens und das Manuskript als Thema beteiligt waren. Mehr nicht. Daraus können wir selbstverständlich weitere Schlüsse über das Manuskript ziehen, die Semantik des Ausdrucks erzwingt dies aber nicht.

Die Differenz tritt umso deutlicher zutage, wenn Chomsky durch einen uns in diesem Zusammenhang unbekannten Eigennamen ersetzt wird.

a. ? Das Manuskript ist von Sandberger zitiert.

b. Das Manuskript ist von Sandberger zitiert worden.

In diesem Fall ist kein Weltwissen abrufbar, das eine geeignete Schlussfolgerung anstoßen könnte, mit was für einer Klasse von Manuskript wir es zu tun haben. Die Perfekt-Variante (15'b) bleibt davon unberührt. Der Satz drückt analog zu (15b) aus, dass es ein Zitierensereignis gab, an dem ein Individuum namens Sandberger als Agens und das Manuskript als Thema beteiligt waren. Die Zustandspassiv-Variante (15'a) hingegen ist in dieser Konstellation pragmatisch abweichend, weil es uns nicht gelingt, auf eine plausible, aus dem Ereignis resultierende Eigenschaft des Manuskripts zu schließen.

Im Folgenden will ich kurz den Kern einer formal-semantischen Modellierung dieses Analysevorschlags für das Zustandspassiv vorstellen; siehe Maienborn (2009). Den Ausgangspunkt bildet die in Maienborn (2003a, 2005) entwickelte Semantik der Kopula. Ein Kopulasatz wie (16) erhält danach die semantische Repräsentation in (17). Der Satz bezeichnet einen Zustand s, in dem die Eigenschaft NEU auf das Manuskript zutrifft. ${ }^{10}$

Das Manuskript ist neu.

$$
\exists \mathrm{s} \text { [NEU (def-manuskript, s)] }
$$

Parallel dazu nehme ich für das Zustandspassiv in (18) die semantische Repräsentation (19) an. Danach bezeichnet (18) einen Zustand, in dem auf das Manuskript eine semantisch unterbestimmte Eigenschaft $Q$ zutrifft,

10 Für die Zwecke des vorliegenden Beitrags nehme ich eine vereinfachte Zustandsanalyse an und gehe nicht auf Einzelheiten zum ontologischen Typ des Zustandsarguments ein (siehe aber Maienborn 2003a, 2005). Ferner gehe ich auch nicht auf eine weitere Differenzierung der beiden Lesarten des Zustandspassivs, Nachzustandslesart (i) und Charakterisierungslesart (ii), ein (siehe hierzu Kratzer 2000; Maienborn 2007). Maienborn (2009) macht einen Vorschlag, wie diese beiden Lesarten als konzeptuelle Spezifizierungen aus der in (20) angegebenen semantischen Struktur abgeleitet werden können.

(i) Das Manuskript ist eingereicht ... Jetzt können wir uns an den Projektantrag machen.

(ii) Das Manuskript ist eingereicht ... aber nicht angenommen.

Ausgeblendet wird ebenfalls der Beitrag des Tempus. 
wobei dieser Zustand als Resultat eines Ereignisses des Manuskript-Einreichens ausgewiesen ist. ${ }^{11}(20)$ gibt den korrespondierenden Beitrag der auf das verbale Partizip anzuwendenden Adjektivierungsoperation an.

(18) Das Manuskript ist eingereicht.

(19) $\exists$ s $\exists$ e $[Q$ (def-manuskript, s) \& RESULT (e, s) \& EINREICH (def-manuskript, e)]

(20) Adjektivierungsoperation:

$\lambda \mathrm{P} \lambda \mathrm{x} \lambda \mathrm{s} \exists \mathrm{e}[\mathrm{Q}(\mathrm{x}, \mathrm{s}) \& \operatorname{RESULT}(\mathrm{e}, \mathrm{s}) \& \mathrm{P}(\mathrm{x}, \mathrm{e})]$

Der Vergleich der beiden semantischen Repräsentationen in (17) und (19) zeigt, dass die Semantik des Zustandspassivs dem Muster regulärer Kopulakonstruktionen folgt. Der Unterschied gegenüber adjektivischen Basisprädikaten besteht lediglich darin, dass das Zustandspassiv dem Subjektreferenten eine intern komplexe und semantisch unterbestimmte Eigenschaft zuweist. Um interpretierbar zu sein, muss die freie Variable $Q$ innerhalb des gegebenen Kontexts einen geeigneten Wert erhalten. Dies kann z.B. über ein abduktives Schlussverfahren à la Hobbs et al. (1993) modelliert werden. Die ökonomischste/beste Spezifizierung der freien Variable Q ist diejenige, die mit den wenigsten kontextuell nicht lizensierten Zusatzannahmen auskommt. Wenn, wie im Falle resultativer Verben, eine entsprechende Eigenschaft bereits lexikalisch eingeführt ist, so ist diese ein bevorzugter Belegungskandidat für Q; siehe (21). Demgegenüber bedarf es eines erheblich größeren pragmatischen Aufwandes, um z.B. für die okkasionelle Bildung schwedentrainiert sein aus (10) eine geeignete Belegung für $\mathrm{Q}$ zu erschließen. Ein entsprechendes Räsonnement könnte etwa als Resultat aus dem Training in Schweden die Eigenschaft, große Mengen Alkohol zu vertragen sprich: Trinkfestigkeit -, ableiten; siehe (22).

(21) a. Der Brief ist geöffnet.

b. $\exists$ s $\exists$ e [Q (def-brief, s) \& RESULT (e, s) \& ÖFFNEN (def-brief, e)] mit $Q=$ OFFEN

(22) a. Der Japaner war schwedentrainiert.

b. $\exists s$ $\exists$ e [Q (def-jap, s) \& RESULT (e, s) \& TRAINIEREN (def-jap, e) $\& \operatorname{IN}(e$, schweden) $]$ mit $Q=$ TRINKFEST

Während also der erforderliche pragmatische Aufwand zur Belegung der freien Variable Q erheblich variieren kann von nahezu vollständiger Vorhersagbarkeit der zu erschließenden Eigenschaft aus dem lexikalischen Gehalt des Basisverbs bis zu massiver Einbeziehung von Kontext und Welt-

$11 \quad$ RESULT (e, s) impliziert, dass der resultierende Zustand s zeitlich unmittelbar an das verursachende Ereignis e anschließt. 
wissen, so ist doch der grundlegende Interpretationsmechanismus derselbe. Dies erklärt die hohe Produktivität und systematisch gegebene Bildbarkeit des Zustandspassivs im Deutschen.

In dem Maße, in dem die zu erschließende Eigenschaft durch die lexikalische Semantik des korrespondierenden Basisverbs festgelegt ist, reduziert sich natürlich der Ad hoc-Charakter des Zustandspassivs. Dies gilt insbesondere für die kausativen Verben wie öffnen, schließen, leeren usw., bei denen $Q$ vollständig lexikalisch determiniert scheint. Allerdings sind selbst hier abweichende kontextuelle Spezifizierungen für Q möglich. Dies zeigen die Beispiele in (23) für geöffnet sein.

(23) a. Die Filmschachtel ist geöffnet, aber wieder zugeklebt.

b. Das Spiel ist geöffnet, aber unbespielt und absolut neuwertig.

Die konzeptuelle Belegung für $Q$ ist hier nicht - wie in (21) - OFFEN, sondern geöffnet sein drückt hier vielmehr aus, dass der Subjektreferent nicht mehr im Originalverpackungszustand ist. Wie (23a) zeigt, kann eine Filmschachtel zugleich geöffnet und zugeklebt (also nicht offen) sein. Bei Ersetzung des Partizips durch das Primäradjektiv offen käme es hingegen zu einem semantischen Widerspruch.

Soweit die Skizze einer formalen Umsetzung der hier vertretenen Zustandspassiv-Analyse: Die Adjektivierung des Partizips bietet ein produktives Ausdrucksmittel für die Konzeptualisierung einer prinzipiell beliebig komplexen, ereignisbasierten Ad hoc-Eigenschaft zur kontextuell induzierten Kategorisierung des Subjektreferenten.

\section{Strukturelle Integration adverbialer Modifikatoren}

Vor dem Hintergrund der oben angestellten Überlegungen zur Interpretation des Zustandspassivs können wir uns nun erneut den adverbialen Modifikatoren zuwenden. Die obigen Ausführungen zu (14) haben schon angedeutet, worin ihr Beitrag besteht, nämlich in einer weitergehenden Spezifizierung und Konturierung der pragmatisch zu erschließenden Ad hocEigenschaft. Was sind die strukturellen Grundlagen hierfür?

Kratzers Analysevorschlag der Integration ereignisbezogener Adverbiale über den Weg der phrasalen Adjektivierung behandelt die betreffenden Modifikatoren in Verbindung mit dem Zustandspassiv als völlig reguläre VP-Modifikatoren wie sie auch in Kombination mit verbalen Partizipien auftreten; vgl. (11b). Die Modifikatoren in (24a)/(25a) sind demnach strukturell identisch mit den Vorgangspassiv-Fällen in (24b)/(25b).

(24) a. Der Brief war mit Wachs versiegelt.

b. Der Brief wurde mit Wachs versiegelt. 
(25) a. Annas Untermieter war von der Polizei gesucht.

b. Annas Untermieter wurde von der Polizei gesucht.

Dies halte ich für falsch. Die beim Zustandspassiv auftretenden adverbialen Modifikatoren sind keine beliebigen VP-Modifikatoren, sondern sie sind prosodisch, syntaktisch und semantisch besonders ausgewiesen. In der Terminologie von Jacobs $(1993,1999)$ sind die betreffenden Modifikatoren in den Verbalkomplex integriert, d.h. sie formen zusammen mit dem Verb eine informationelle Einheit. Für klassische VP-Modifikatoren gilt dies nicht. Diese kompakte informationelle Einheit aus Partizip und Modifikator ist die Voraussetzung dafür, dass der Modifikator von der Adjektivierung erfasst werden und einen Beitrag zur Ableitung einer komplexen Ad hoc-Eigenschaft leisten kann, und sie charakterisiert den hier aufscheinenden Übergangsbereich zwischen Wort und Phrase.

Im Weiteren werde ich Daten aus dem Bereich von Prosodie, Syntax und Semantik vorstellen, die Hinweise auf diesen besonderen strukturellen Status der Verbindung von Partizip und Modifikator beim Zustandspassiv in Abgrenzung zum Vorgangspassiv liefern, und die den für das Zustandspassiv charakteristischen Strukturausbau zwischen Wort und Phrase weiter beleuchten.

\subsection{Prosodische Integration}

Betrachten wir zunächst die Prosodie. Beim Zustandspassiv wird neutraler Satzakzent mittels Hauptakzent auf dem verbadjazenten Modifikator gekennzeichnet; siehe (26a). ,Mit Wachs versiegelt sein' wird dabei als eine kompakte Informationseinheit präsentiert. Hauptakzent auf dem Partizip - und damit ,,informationelle Separierung" (Jacobs 1999) des Modifikators ist abweichend bzw. erfordert starken Kontrastfokus; siehe (26b). Zieht man zum Vergleich die Vorgangspassiv-Variante heran, so wird hier neutraler Satzakzent am Partizip gekennzeichnet; siehe (27b). Der Vorgang des Versiegelns und das dabei eingesetzte Wachs werden als zwei unabhängige Informationseinheiten dargestellt. Hauptakzent auf dem Modifikator, wie in (27a), ist nur mit Kontrastfokus vereinbar. (Hauptakzent wird durch Fettdruck angezeigt.)

(26) Anna sah sofort, ...

a. dass der Brief an Leo [w mit Wachs versiegelt war] neutraler Satzakzent

b. ?? dass der Brief an Leo ${ }_{\text {VP }}$ mit Wachs versiegelt war] (und nicht verschmiert) 
(27) Anna sah sofort, ...

a. dass der Brief an Leo [r gerade mit Wachs versiegelt wurde] (statt mit Siegellack)

b. dass der Brief an Leo [r gerade mit Wachs versiegelt wurde] neutraler Satzakzent

Die Beispiele (28) und (29) zeigen dasselbe Muster bei Agens-Angaben:

(28) Anna vermutete, ...

a. dass ihr Untermieter [ von der Polizei gesucht war] neutraler Satzakzent

b. ?? dass ihr Untermieter [ ${ }_{v}$ von der Polizei gesucht war] (und nicht versteckt)

(29) Anna vermutete, ...

a. dass ihr Untermieter [grade von der Polizei gesucht wurde] (und nicht von der Mafia)

b. dass ihr Untermieter [r gerade von der Polizei gesucht wurde] neutraler Satzakzent

Während adverbiale Modifikatoren beim Zustandspassiv also mit dem Partizip eine informationelle Einheit bilden müssen, ist dies beim Vorgangspassiv nicht der Fall. Die in (26)-(29) sichtbar werdenden prosodischen Unterschiede liefern erste Evidenz dafür, dass die betreffenden Modifikatoren strukturell nicht identisch sind.

Betrachten wir weiterhin Daten wie (30), die vom Typ der in der Literatur als nicht wohlgeformt bewerteten Modifikationsfälle sind; siehe die Diskussion zu (5).

a. ?? Die Kartoffeln sind von Otto gekauft.

b. ?? Das Manuskript ist mit der Leselupe gelesen.

Wird, wie in (31), durch die prosodische Struktur eine Komplexbildung angezeigt, so zwingt uns die Grammatik geradezu, eine geeignete pragmatische Deutung zu finden - in Gestalt einer aus dem gegebenen Ereignis unter Rekurs auf Kontext und Weltwissen abgeleiteten, plausiblen Ad hocEigenschaft.

(31) a. Die Kartoffeln sind von Otto gekauft. (Die schmecken bestimmt lecker, denn Otto versteht was von Kartoffeln.)

b. Das Manuskript ist mit der Leselupe gelesen. (Da ist kein Tippfehler mehr drin.)

Die Sätze in (30) würde man out of the blue vermutlich als nicht wohlgeformt zurückweisen. Wenn die Prosodie allerdings die Integration des Modifikators anzeigt, wie in (31), dann lassen sich selbst Ausdrücke wie von Otto gekanft 
sein oder mit der Leselupe gelesen sein als sinnvolle Ad hoc-Eigenschaftszuschreibungen an den Subjektreferenten deuten, etwa entlang der in Klammern hinzugefügten kontextuellen Explizierungen. Das Fazit hieraus ist, dass Sätze wie (30) oder (5) keineswegs als grammatisch nicht wohlgeformt zu bewerten sind, sondern - die strukturelle Integration des Modifikators vorausgesetzt - allenfalls einen höheren pragmatischen Aufwand zur Erschließung einer plausiblen Ad hoc-Eigenschaft erfordern. Die genuin grammatischen Kombinationsbeschränkungen für adverbiale Modifikatoren beim Zustandspassiv erweisen sich damit als viel liberaler und weniger idiosynkratisch als dies der erste Augenschein vermuten lässt. ${ }^{12}$

\subsection{Syntaktische Integration}

Wenden wir uns als nächstes der syntaktischen Evidenz für die unterschiedliche strukturelle Einbindung adverbialer Modifikatoren beim Zustandspassiv gegenüber dem Vorgangspassiv zu. Wenn die ereignisbezogenen Modifikatoren beim Zustandspassiv eine besonders enge strukturelle Bindung mit dem verbalen Partizip eingehen, dann ist zu erwarten, dass sie sich, anders als ihre Pendants beim Vorgangspassiv, durch eine gewisse Ortsfestigkeit auszeichnen. Die folgenden Daten zur Vorfeldbesetzung und zum Scrambling bestätigen diese Vorhersage.

(32) a. Die Erdbeeren sind / wurden im Bioladen gekauft.

b. Im Bioladen wurden die Erdbeeren gekauft.

c. ?? Im Bioladen sind die Erdbeeren gekauft.

(33) a. Das Lamm-Curry ist / wird mit Ingwer abgeschmeckt.

b. Mit Ingwer wird das Lamm-Curry abgeschmeckt.

c. ?? Mit Ingwer ist das Lamm-Curry abgeschmeckt.

(34) a. Oliver ist / wurde von Zsa Zsa Gabor adoptiert.

b. Von Zsa Zsa Gabor wurde Oliver adoptiert.

c. ?? Von Zsa Zsa Gabor ist Oliver adoptiert.

Während eine Topikalisierung des Lokal-, Instrumental- oder Agensadverbials beim Vorgangspassiv ohne besondere (konstruktionsspezifische) Auflagen ${ }^{13}$ möglich ist (siehe die b.-Varianten in (32)-(34)), führt im Falle des Zustandspassivs eine Herauslösung des betreffenden Adverbials aus seiner VP-internen Basisposition zu einer grammatisch markierten Struktur. Die c.-Varianten in (32)-(34) sind nur bei klarer Kontrastakzentsetzung z.B. auf

12 Zu den vergleichsweise wenigen grammatischen Ausschlusskriterien für die Bildbarkeit des Zustandspassivs siehe Maienborn (2007, S. 102 ff.).

13 Bezüglich der für die Vorfeldbesetzung generell geltenden informationsstrukturellen Implikationen siehe Frey $(2004,2006)$. 
dem Adverbial zulässig. ${ }^{14}$ Dies zeigt, dass es sich um markierte Strukturen handelt, die allenfalls über den letzten Ausweg Kontrast gerettet werden können. Die Adverbiale beim Vorgangspassiv sind hingegen nicht auf solch massive kontrastive Stützung angewiesen.

Ein analoger Befund ergibt sich beim Vergleich des Scramblingverhaltens der Modifikatoren. Als obligatorisch in den Verbalkomplex integrierte Elemente lassen sich die beim Zustandspassiv auftretenden ereignisbezogenen Adverbiale nur schlecht scrambeln; für ihre nicht-integrierten Pendants beim Vorgangspassiv gilt dies nicht. (35)-(38) illustrieren das Verhalten von Instrumentaladverbialen, (39)-(42) das von durch-PPn. (Zum Ereignisbezug von durch-Adverbialen siehe Solstad 2007.)

(35) Maria hat gesagt, ...

a. dass der Weg mit Mosaiksteinen gefliest wird.

b. dass mit Mosaiksteinen der Weg gefliest wird.

(36) Maria hat gesagt, ...

a. dass der Weg mit Mosaiksteinen gefliest ist.

b. ?? dass mit Mosaiksteinen der Weg gefliest ist.

(37) Der Dozent hat bemerkt, ...

a. dass die Tafel mit einem Edding beschmiert wird.

b. dass mit einem Edding die Tafel beschmiert wird.

(38) Der Dozent hat bemerkt, ...

a. dass die Tafel mit einem Edding beschmiert ist.

b. ?? dass mit einem Edding die Tafel beschmiert ist.

(39) Die Sekretärin hat angedeutet, ...

a. dass der Politiker durch Drohanrufe verunsichert wird.

b. dass durch Drohanrufe der Politiker verunsichert wird.

(40) Die Sekretärin hat angedeutet, ...

a. dass der Politiker durch Drohanrufe verunsichert ist.

b. ?? dass durch Drohanrufe der Politiker verunsichert ist.

(41) Der Trainer hat versichert, ...

a. dass die Skifahrerin durch einen Helm geschützt wird.

b. dass durch einen Helm die Skifahrerin geschützt wird.

(42) Der Trainer hat versichert, ...

a. dass die Skifahrerin durch einen Helm geschützt ist.

b. ? dass durch einen Helm die Skifahrerin geschützt ist.

14 Oder aber es stellt sich eine Listeninterpretation ein; vgl. (1) für (33c):

(i) Mit Ingwer ist das Lamm-Curry abgeschmeckt, mit Koriander das Huhn, ... 
Die in (35)-(42) angegebenen Bewertungen wurden im Rahmen einer Beurteilungsstudie überprüft und empirisch erhärtet (siehe Gese/Stolterfoht 2010). Während die Testsätze in der Abfolge Subjekt < Modifikator in der Vorgangspassiv- und der Zustandspassiv-Version von den Probanden als gleich gut bewertet wurden, zeigt sich in der gescrambelten Abfolge Modifikator < Subjekt ein deutlicher Unterschied: Die gescrambelte Abfolge wird beim Zustandspassiv signifikant schlechter bewertet als beim Vorgangspassiv. Dies kann als weiterer Hinweis darauf gewertet werden, dass der strukturelle Status des Modifikators beim Zustandspassiv ein anderer ist als beim Vorgangspassiv.

\subsection{Semantische Integration}

Semantische Reflexe der Bildung einer kompakten Informationseinheit aus Modifikator und Partizip beim Zustandspassiv zeigen sich z.B. im Hinblick auf die anaphorische Wiederaufnahme. Durch die Integration in den Verbalkomplex verliert der Modifikator seine referenzielle Unabhängigkeit und ist als Teil des Prädikats nicht mehr in vollem Umfang referenziell zugänglich. Der Vergleich der Verhältnisse beim Zustandspassiv mit denen beim Vorgangspassiv einerseits und denen entsprechender Komposita andererseits erhellt den Übergangscharakter der Zustandspassivkonstellation zwischen Wort und Phrase: Im Vorgangspassiv-Fall (43a), vom TÜV geprïft werden, bildet der Agens eine eigenständige, referenziell unabhängige Informationseinheit, die im Folgenden anaphorisch wiederaufgenommen werden kann. Beim Kompositum TUV-gepriff in (43c) hingegen ist der Agens vollständig in das Wort integriert und damit referenziell nicht mehr zugänglich. Der Zustandspassiv-Fall (43b), vom TÜV geprïft sein, ist zwischen diesen beiden Extremen angesiedelt: Die anaphorische Wiederaufnahme ist zwar nicht vollständig blockiert, wie bei (43c), allerdings ist sie auch nicht völlig reibungslos möglich wie in (43a); vielmehr führt der Versuch einer anaphorischen Wiederaufnahme zu einem Konflikt, der allenfalls partiell durch die nachträgliche Rekonstruktion eines entsprechenden Diskursrefenten gelöst werden kann.

(43) a. Mein Auto ist vom TüV geprüft worden. Er hatte nichts zu beanstanden.

b. Mein Auto ist vom TÜV geprüft. ? Er hatte nichts zu beanstanden.

c. Mein Auto ist TÜV-geprüft. * Er hatte nichts zu beanstanden.

Mit der sukzessiven Aufgabe der referenziellen Eigenständigkeit korrespondiert eine weitere semantische Beobachtung. So besagt die Interpretation von (43a) lediglich, dass das Auto der Sprecherin Gegenstand einer 
vom TÜV durchgeführten Prüfung war. (43a) ist damit mit der Annahme kompatibel, dass diese Prüfung negativ ausfiel; siehe (44a). Dies ist im Falle des Kompositums ausgeschlossen. Aus dem Zutreffen des komplexen Prädikats TUV -geprifft folgt, dass die Prüfung bestanden wurde; (44c) ist damit semantisch widersprüichlich (gekennzeichnet mit \#).

(44) a. Mein Auto ist vom TÜV geprüft worden. Leider ist die Prüfung negativ ausgefallen.

b. ?? Mein Auto ist vom TÜV geprüft. Leider ist die Prüfung negativ ausgefallen.

c. \# Mein Auto ist TÜV-geprüft. Leider ist die Prüfung negativ ausgefallen.

Was ist mit der Zustandspassiv-Konstellation (43b)? Auch hier gibt es eine starke Präferenz für die Folgerung, dass die Prüfung erfolgreich absolviert wurde; siehe (44b). Ich würde dennoch dafür plädieren, dieser Schlussfolgerung den Status einer konversationellen Implikatur einzuräumen, die - bei allerdings nur massiver kontextueller Stützung - aufhebbar ist. Ein solcher Kontext könnte z.B. eine groß angelegte Evaluation sein, bei der der TÜV selbst auf dem Prüfstand steht und gegen eine konkurrierende PrüferOrganisation antritt. In einem solchen kontrastiven Setting ließe sich sagen: Diese Autos sind vom TÜV gepriff, ohne damit zu implizieren, dass die Prüfung in jedem Fall erfolgreich verlief. Hingegen würde die Aussage Diese Autos sind TÜ -gepriff auch in diesem Kontext weiterhin nur die Klasse der vom TÜV positiv getesteten Wagen identifizieren. Auch für die konzeptuellbasierten Schlussfolgerungen gilt somit - wie oben bereits für die lexikalisch-semantisch induzierten Schlüsse am Beispiel des kausativen Verbs öffnen diskutiert - je stärker sie kontextübergreifend mit dem jeweiligen Ereigniskonzept assoziiert sind (etwa: PRÜFEN - PRÜFUNG-BESTEHEN), desto weniger ad hoc erscheint die erschlossene Eigenschaft.

Die in Abschnitt 4 entwickelte Semantik des Zustandspassivs kann die obige Beobachtung unmittelbar erfassen. Zunächst einmal geht sie mit der Forderung nach einer semantisch unterbestimmten Eigenschaft, die als Resultat des von dem Verb bezeichneten Ereignisses ausgewiesen ist, über das betreffende Ereignis hinaus. Anders als im Vorgangspassivfall reicht es also bei (43b)/(44b) nicht, dass das Auto Gegenstand einer TÜV-Prüfung war. Vielmehr muss eine daraus resultierende, kontextuell saliente Eigenschaft erschlossen werden, und zwar unter geringstmöglichem pragmatischen Aufwand. Dies ist im Default-Fall die an den erfolgreichen Abschluss der Prüfung gebundene Verkehrstauglichkeit. Nur sehr spezielle Kontexte, wie etwa der oben geschilderte, legen eine andere Instantiierung der Eigenschaftsvariable nahe. Der (mehr oder weniger ausgeprägte) Ad hoc-Charakter der Eigenschaftszuschreibung beim modifizierten Zustandspassiv 
ist damit in der kompositional angelegten semantischen Unterbestimmtheit verankert und setzt auf der engen strukturellen Bindung zwischen Modifikator und Partizip auf.

Ich will die Liste der Evidenzen für eine strukturelle Integration adverbialer Modifikatoren beim Zustandspassiv mit dem Hinweis auf ein psycholinguistisches Experiment abschließen, welches die Frage der referenziellen Eigenständigkeit bzw. Integration des Modifikators zum Gegenstand hat (siehe Gese/Stolterfoht 2010). Es handelt sich um eine an Ratcliff/McKoon (1978) angelehnte Reaktionszeitstudie mit Wortwiedererkennungsaufgabe, die von der Annahme ausgeht, dass integrierte Elemente schneller wiedererkannt werden, wenn sie nach dem integrierenden Element präsentiert werden. Konkret geht es um die Frage, ob der Modifikator beim Zustandspassiv, nicht aber beim Vorgangspassiv, durch das zuvor präsentierte Partizip „gebahnt" wird (semantisches Priming). Die Versuchspersonen bekamen in diesem Experiment Vorgangs- und Zustandspassivsätze vom Typ (45a) präsentiert und mussten im Anschluss für zwei aufeinander folgende Wörter jeweils entscheiden, ob diese im zuvor gelesenen Satz vorkamen. In den relevanten Bedingungen waren dies zunächst das Partizip und darauf folgend entweder das Modifikator-Nomen oder das Subjekt-Nomen; siehe (45b). Unsere Hypothesen waren: (H1) eine schnellere Wiedererkennung des Modifikator-Nomens nach dem Zustandspassivsatz und (H2) keine Reaktionszeitunterschiede zwischen Vorgangspassiv und Zustandspassiv bei der Wiedererkennung des Subjekt-Nomens; siehe (45c).

(45) Reaktionszeitstudie mit Wortwiedererkennungsaufgabe (Gese/Stolterfoht 2010)

a. Testsätze:

(ZP) Der Weg ist mit Mosaiksteinen gefliest.

(VP) Der Weg wird mit Mosaiksteinen gefliest.

b. Aufgabe: „Kam das Wort im zuvor gelesenen Satz vor?" (mod) GEFLIEST [ja/nein] MOSAIKSTEINE [ja/nein] (subj) GEFLIEST [ja/nein] WEG [ja/nein]

c. Hypothesen:

H1: $\quad$ ZPmod $<\operatorname{vPmod}$

H2: $\quad$ ZPsubj $=$ VPsubj

Beide Hypothesen wurden bestätigt. Es zeigte sich eine signifikant schnellere Wiedererkennung des Modifikator-Nomens in der Zustandspassiv-Bedingung gegenüber der Vorgangspassiv-Bedingung. Hingegen zeigten sich keine signifikanten Reaktionszeitunterschiede für das Subjekt-Nomen. Aus diesen Ergebnissen lässt sich schließen, dass das Modifikator-Nomen beim 
Zustandspassiv (und nur dort) durch das Partizip semantisch gebahnt wird; siehe Gese/Stolterfoht (2010) zu weiteren Details zu Durchführung des Experiments, Vorstudien zum Satzmaterial und statistischer Auswertung.

Die Befunde wurden durch eine weitere Kontrollstudie erhärtet, bei der mit identischem Testmaterial lediglich die Wiedererkennung von Modifikator- oder Subjekt-Nomen ohne vorherige Präsentation des Partizips getestet wurde; siehe (46b). Hier erwarten wir keine Reaktionszeitunterschiede für das Modifikator-Nomen (H3); siehe (46c). Auch diese Hypothese wurde bestätigt.

(46) Kontrollstudie

a. Testsätze:

(ZP) Der Weg ist mit Mosaiksteinen gefliest.

(VP) Der Weg wird mit Mosaiksteinen gefliest.

b. Aufgabe: „Kam das Wort im zuvor gelesenen Satz vor?"

(mod) MOSAIKSTEINE [ja/nein]

(subj) WEG [ja/nein]

c. Hypothesen:

H3: $\quad$ ZPmod $=$ VPmod; ZPsubj $=$ VPsubj

Die Ergebnisse der Kontrollstudie unterstützen damit die Deutung des beobachteten Effekts in der ersten Reaktionszeitstudie als Priming-Effekt: Beim Zustandspassiv wird das integrierte Element (= Modifikator-Nomen) durch das integrierende Element (= Partizip) semantisch gebahnt. Dies zeigt, dass Modifikator und Partizip beim Zustandspassiv eine besonders enge Bindung eingehen. Im Falle des Vorgangspassivs kommt es hingegen zu keiner entsprechenden Bahnung. Dies ist mit der Annahme kompatibel, dass in diesem Fall reguläre VP-Modifikatoren vorliegen.

\section{Fazit}

Die oben präsentierten Daten wurden mit verschiedenen Methoden gewonnen: Introspektion, Beurteilungsstudien, experimentelle Studien; und sie betreffen verschiedene Ebenen der grammatischen Strukturbildung: Prosodie, Syntax, Semantik. Somit ist eine breite empirische Fundierung für theoretische Schlussfolgerungen gegeben. Die Befunde zeigen übereinstimmend, dass die strukturelle Einbindung ereignisbezogener Modifikatoren beim Zustandspassiv nicht identisch mit derjenigen beim Vorgangspassiv ist. Sie widerlegen damit Kratzers Ausgangshypothese für ihren Vorschlag der Einbeziehung der betreffenden Modifikatoren über phrasale Adjekti- 
vierung; siehe die Diskussion zu (11) und (24)/(25). Vielmehr unterstützen die Befunde die Annahme einer besonderen strukturellen Nähe von Partizip und Modifikator beim Zustandspassiv. Beide bilden gemeinsam ein komplexes Prädikat, aus dem qua Adjektivierungsoperation eine resultierende Ad hoc-Eigenschaft abgeleitet wird; siehe (20).

Ich will an dieser Stelle auf einen Punkt in der Analyse von Schlücker (2005) noch etwas näher eingehen. Schlücker folgt im Wesentlichen dem hier vertretenen Ansatz einer verbnahen Platzierung der betreffenden Modifikatoren und entwickelt auf dieser Grundlage eine konzeptuelle Analyse ereignisbezogener Modifikatoren beim Zustandspassiv im Rahmen von Barsalous (1992) „Frame“-Konzept. Allerdings stellt sie für die Klasse der von-Adverbiale fest, dass es hier neben den obligatorisch in den Verbalkomplex integrierten von-PPn einen zweiten Typus von von-PPn gibt, der nicht dem in Abschnitt 5 geschilderten prosodischen und syntaktischen Muster folgt, sondern sich wie die beim Vorgangspassiv auftretenden regulären VPModifikatoren verhält. Schlückers Musterfall ist (47a). Neutraler Satzakzent etwa wird hier nicht auf dem Modifikator, sondern auf dem Partizip realisiert; siehe (47b) vs. (47c) sowie Schlückers weitere syntaktische Indizien.

(47) a. Peter ist von dem Gejammer genervt.

b. weil Peter von dem Gejammer genervt ist. nur kontrastiv

c. weil Peter von dem Gejammer genervt ist. neutraler Satzakzent

Als Erklärung für diesen Befund greift Schlücker auf Kratzers Mechanismus der phrasalen Adjektivierung zurück, den sie allerdings allein für entsprechende von-PPn reserviert. Ich folge ihr in diesem Schritt nicht und vertrete vielmehr die Auffassung, dass Schlückers von-PPn vom Typ (47a) keine Agens-Angaben sind und auch keinen anderweitigen Ereignisbezug haben, sondern in Solstads (2007) Redeweise einen Grund als kausal involvierten Sachverhalt benennen (siehe auch Solstad 2010). Die betreffenden Modifikatoren haben folglich keinen Ereignisbezug, sondern Sachverhaltsbezug und sind entsprechend strukturell höher angesiedelt. In der Tat finden sich derartige Kausalangaben nicht nur beim Zustandspassiv, sondern auch bei einfachen prädikativen Adjektiven:

(48) a. Paul war von der Reise müde.

b. Die Straße war vom Regen nass.

c. Der Rasen war von dem Rumgerenne platt.

d. Ihr Gesicht war von der Sonne rot.

Das heißt, die von-Modifikatoren vom Typ (47) liegen außerhalb des Skopus der Adjektivierungsoperation; es handelt sich, gleich den (48)-Fällen, um Modifikatoren des Kopula-Prädikativ-Komplexes. Mit dem Zustandspassiv im engeren Sinne haben sie nichts zu tun. ${ }^{15}$

Ich danke Torgrim Solstad für unsere Diskussion dieser Daten. 
Zurück zum Zustandspassiv. Wie soll das spezifische Strukturverhältnis zwischen Partizip und Modifikator syntaktisch umgesetzt werden? Schaut man sich nach möglichen Vorbildern um, so sind ähnliche Formen der Komplexbildung aus anderen Zusammenhängen und Sprachen wohlbekannt siehe etwa die durch Baker (1988) angestoßene Inkorporationsdiskussion sowie die Überlegungen zum Verbalkomplex im Deutschen z.B. bei Bierwisch (1988), Steinitz (1989), Frey/Tappe (1992), Frey (1993) und Haider (1993, 2010). In zweierlei Hinsicht weicht die hier betrachtete Konstruktion von klassischen Inkorporationsfällen ab. Erstens sind die integrierten Elemente in unserem Fall klare Modifikatoren, und keine Argumente des verbalen Kopfs, für die Bakers Inkorporationstheorie - und im Übrigen auch Jacobs' Theorie der Integration - ausgelegt ist. ${ }^{16}$ Und zweitens sind die integrierten Elemente eindeutig phrasale Konstituenten.

Dies genau ist die Crux für eine geradlinige strukturelle Umsetzung der obigen Befunde. Naheliegend nach dem bisher Gesagten wäre eine Modellierung über Kopf-Adjunktion wie in (49) (siehe den Vorschlag in Maienborn 2007).$^{17}$ Dies erfasst die unmittelbare strukturelle Nähe zwischen Modifikator und Partizip, und es erlaubt, für das Zustandspassiv eine alleinige, auf lexikalischer Ebene angesiedelte Adjektivierungsoperation anzunehmen. Strukturell höher angesiedelte VP-Modifikatoren werden von der lexikalischen Adjektivierung nicht erfasst und sind deshalb beim Zustandspassiv ausgeschlossen.

16 Jacobs (2004) erwägt für die hier in Rede stehenden ereignisbezogenen Modifikatoren - in seiner Terminologie „Zirkumstantien“ - eine Neubewertung als Argumente. Damit ließe sich, angewandt auf unseren Fall, ihre Integration in den Verbalkomplex unter Beibehaltung der ursprünglichen Argumentrestriktion erfassen. Allerdings scheint mir eine solche Aufgabe der klassischen Argument/Modifikator-Unterscheidung zu weitreichend zu sein. Es sind in unserem Fall auch nicht Zirkumstantien per se, die als Integrationskandidaten in Frage kommen, sondern nur solche in besonderer struktureller Nähe zum Verb. Im Zusammenhang mit einer Analyse lokaler Modifikatoren habe ich in Maienborn $(1996,2001)$ argumentiert, dass die verbnah, unterhalb des direkten Arguments basisgenerierten Modifikatoren vom Typ auf den Fingern pfeifen, in der Pfanne braten, an den Füßen kitzoeln grammatisch den Status von Adjunkten haben; die vorgeschlagene semantische Analyse läuft aber darauf hinaus, ihnen konæeptuell durchaus Argumentstatus zuzubilligen: Sie aktivieren und füllen funktional eingebundene Slots innerhalb der konzeptuellen Ereignisstruktur, ohne dass dies allerdings Auswirkungen auf die lexikalisch fixierte Argumentstruktur der involvierten Verben hätte (siehe hierzu insbesondere die konzeptuelle Detailanalyse in Maienborn 2003b). Die betreffenden Ausdrücke - dies gilt für die verbnahen lokalen Modifikatoren ebenso wie für die hier besprochenen ereignisbezogenen Adverbiale beim Zustandspassiv - sind demnach Zwitter: Grammatisch handelt es sich um frei hinzutretende Modifikatoren; konzeptuell greifen sie tief in die Ereignisstruktur ein und bilden gemeinsam mit dem Verb ein komplexes Prädikat. Unter dieser Perspektive wären dann allerdings die strukturellen Voraussetzungen für das Zustandekommen von Integration entsprechend zu liberalisieren.

17 Siehe auch Haiders (2010, S. 335 ff.) aktuellen Vorschlag zur Syntax von V0-Clustern als $\mathrm{X}^{0}$-Adjunktionsstrukturen, in den er die Einbindung von so genannten „co-predicates“ (insbesondere resultative und direktionale Prädikate) explizit einbezieht. 
(49) Strukturelle Integration via Kopf-Adjunktion

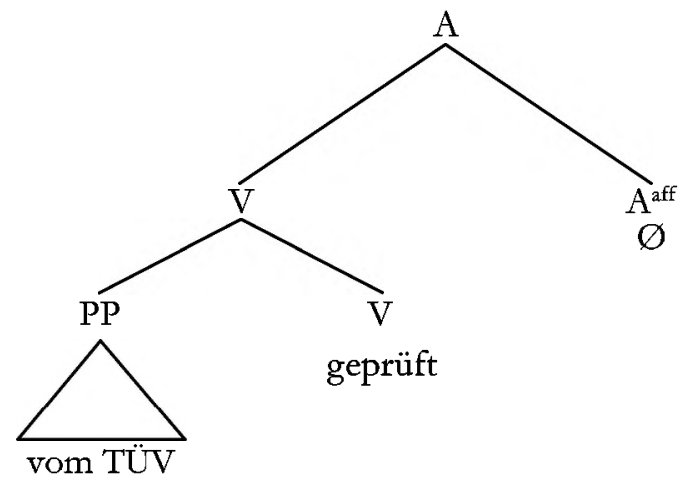

Das Lexikon wäre damit weiterhin als Domäne für morphologische Operationen, wie eben auch den hier diskutierten lexikalischen Kategorienwechsel beim Partizip, ausgewiesen, und das mächtige Instrument der phrasalen Adjektivierung wäre verzichtbar. Der Preis dafür allerdings ist, dass wir mit (49) behaupten, der komplexe Ausdruck vom TÜV geprïf sei ein Wort. Dies ist er nach unserem Standardverständnis von Wörtern und ihrer Abgrenzung gegenüber Syntagmen sicher nicht. Die Beiträge in diesem Band zeigen allerdings, dass eine solche Abgrenzung und eine entsprechend disjunkte Einteilung, ,Wort oder Syntagma?" bei allem heuristischen Nutzen nicht haltbar ist (siehe insbesondere den Beitrag von Jacobs). Jacobs' Liste von Übergangserscheinungen zwischen Wort und Syntagma wäre nach dem hier Gesagten also um einen weiteren Ausdruckstypus zu ergänzen, der zwar auf syntaktischer Komplexbildung beruht, dessen interne Struktur jedoch für bestimmte syntaktische und semantische Prozesse nicht mehr zugänglich ist.

(50) $\mathrm{X}^{\prime}$ als Integrationsdomäne

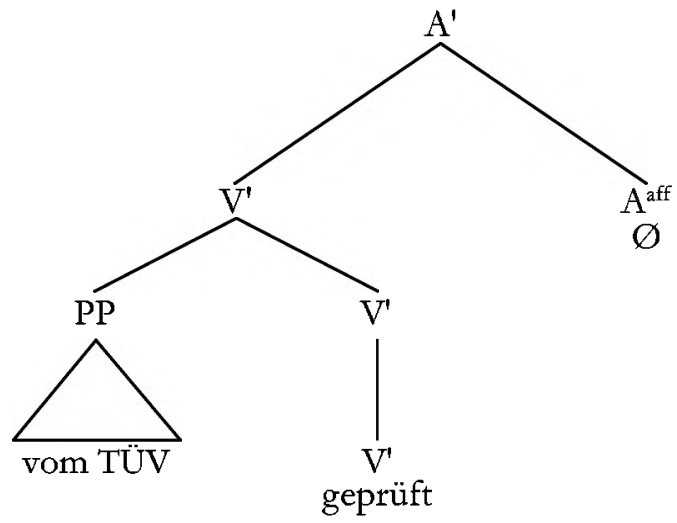


Sicher sind Alternativen zu (49) denkbar, etwa im Sinne von Frey/Tappe (1992) und Frey (1993) die Reservierung einer Zwischenprojektionsstufe X' als die relevante Domäne für Integration, innerhalb derer eine besondere strukturelle Nähe zum Kopf besteht. Nicht-integrierte Argumente und die Standard-Modifikatoren sind in diesem Modell auf VP-Ebene angesiedelt. Damit wäre $V^{\prime}$ als die strukturell höchste Position auszuweisen, auf der die Adjektivierung verbaler Partizipien (samt integrierter Modifikatoren) erfolgen kann; siehe (50).

Ob eine solche $\mathrm{X}^{\prime}$-Zwischenprojektion als strukturelles Auffangbecken für diese und andere Grenzerscheinungen zwischen Wort und Syntagma sich letztlich als tragfähig und nützlich erweist, bleibt zu zeigen - ebenso wie Theorieansätze, die von vorneherein die Unterscheidung zwischen Lexikon und Syntax aufgeben, eine Erklärung für die in Abschnitt 5 gezeigten Unterschiede der Modifikatoren bei Zustandspassiv und Vorgangspassiv bieten müssen.

Die genauen strukturellen Grundlagen für die im Grenzbereich von Wort- und Phrasenstruktur angesiedelte Form der Komplexbildung bedürfen dringend weiterer Klärung. Allerdings will ich abschließend auch unterstreichen, dass der hier vorgestellte Ansatz eine strikt kompositionale Semantik für die ereignisbezogenen Modifikatoren beim Zustandspassiv bietet, die mit beiden Strukturoptionen (49) und (50) (oder möglichen Varianten) kompatibel ist. Dies wird in (51) noch einmal exemplarisch durchgespielt. Ich lege dabei zur leichteren Lesbarkeit eine neo-davidsonsche Darstellung der betreffenden Lexikoneinträge mittels thematischer Rollen zugrunde; siehe $(51 \mathrm{a} / \mathrm{b})$. Verbnah platzierte Modifikatoren werden entsprechend der Davidsonschen Grundeinsicht als zusätzliche Prädikate über den verbalen Ereignisreferenten konjunktiv angeschlossen $(51 \mathrm{c}){ }^{18}$

(51) Das Auto ist vom TÜV geprüft.

a. geprüft: $\quad \lambda y \lambda$ e [PRÜF (e) \& THEMA (y, e)]

b. vom TÜV: $\lambda e^{\prime}[$ AGENS (TÜV, e')]

c. $[\mathrm{v}$ vom TÜV geprüft]: $\lambda y$ ye [PRÜF (e) \& THEMA (y, e) \& AGENS (TÜV, e)]

Die in (51c) angegebene verbale Struktur fällt unter den Skopus des in (20) vorgeschlagenen Adjektivierungsoperators, hier wiederholt in (51d). Das Resultat der Anwendung des Adjektivierungsoperators auf (51c) ist in (51e) angegeben.

$$
\begin{aligned}
& \text { d. } A^{\text {Aff: }} \quad \lambda P \lambda x \lambda s \exists \text { e }[\mathrm{Q}(\mathrm{x}, \mathrm{s}) \& \operatorname{RESULT}(\mathrm{e}, \mathrm{s}) \& \mathrm{P}(\mathrm{x}, \mathrm{e})] \\
& \text { e. [A [v vom TÜV geprüft]]: } \lambda \mathrm{x} \lambda \mathrm{s} \exists \mathrm{e}[\mathrm{Q}(\mathrm{x}, \mathrm{s}) \& \operatorname{RESULT}(\mathrm{e}, \mathrm{s}) \& \\
& \text { PRÜF (e) \& THEMA (x, e) \& AGENS (TÜV, e)]] }
\end{aligned}
$$

\footnotetext{
18 Die verantwortliche Kompositionsregel für die Verbindung des semantischen Beitrags des Modifikators $P$ mit dem des Modifikanden $Q$ ist in (i) angegeben.

(i) $\lambda \mathrm{P} \lambda Q \lambda e[Q(e) \& P(e)]$
} 
Das Resultat (51e) wird als komplexes Prädikat in die Kopula eingespeist $(51 \mathrm{f} / \mathrm{g})$.

\author{
f. Kopula: $\lambda \mathrm{P} \lambda \mathrm{x} \lambda \mathrm{s}[\mathrm{P}(\mathrm{x}, \mathrm{s})]$ \\ g. vom TÜV geprüft sei-: $\lambda \mathrm{x} \lambda \mathrm{s}$ ヨe [Q $(\mathrm{x}, \mathrm{s}) \& \operatorname{RESULT}(\mathrm{e}, \mathrm{s}) \&$ \\ PRÜF (e) \& THEMA (x, e) \& AGENS (TÜV, e)]]
}

Nach Sättigung des Subjektarguments $(51 \mathrm{~h} / \mathrm{i})$ und existentieller Bindung des Zustandsarguments (via Finitheit) ergibt sich als kompositional-semantische Struktur (51j).

h. das Auto: DEF x [AUTO (x)]

abgekürzt mit: a

i. das Auto vom TÜV geprüft sei-: $\lambda \mathrm{s}$ ヨe $[\mathrm{Q}(\mathrm{a}, \mathrm{s}) \& \operatorname{RESULT}(\mathrm{e}, \mathrm{s})$ \& PRÜF (e) \& THEMA (a, e) \& AGENS (TÜV, e)]l

j. Das Auto ist vom TÜV geprüft: (Kompositionale Semantik) $\exists$ s $\exists$ e [Q (a, s) \& RESULT (e, s) \& PRÜF (e) \& THEMA (a, e) \& AGENS (TÜV, e)]]

In Prosa besagt (51j), dass es einen Zustand s gibt, in dem die unterbestimmte Eigenschaft $Q$ auf ein im Diskurs eindeutig identifizierbares Auto a zutrifft. Dieser Zustand ist als Result eines Prüfereignisses e ausgewiesen, an welchem der TÜV als Agens und das Auto als Gegenstand der Prüfung beteiligt waren. Eine plausible konzeptuelle Spezifikation für Q folgt in (51k). Danach wird die kompositional unterbestimmt verbliebene Eigenschaft $Q$ als Fahrtauglichkeit identifiziert. ${ }^{19}$ In Prosa: Das im Diskurs eindeutig identifizierbare Auto a befindet sich, resultierend aus einer Prüfung durch den TÜV, im Zustand der Fahrtauglichkeit.

$$
\begin{aligned}
& \text { k. Das Auto ist vom TÜV geprüft: (Konzeptuelle Spezifikation) } \\
& \text { ヨs ヨe [FAHRTAUGLICH (a, s) \& RESULT (e, s) \& PRÜF (e) \& } \\
& \text { THEMA (a, e) \& AGENS (TÜV, e)]] }
\end{aligned}
$$

Die in der Adjektivierungsoperation kompositional angelegte semantische Unterbestimmtheit, vermittelt über die freie Variable Q, ist es, die die für das Zustandspassiv charakteristische Einbeziehung von Kontext und Weltwissen ermöglicht und die Ableitung einer kontextuell salienten Ad hoc-Eigenschaft sicherstellt. Auf diese Weise ergibt sich als Ergebnis der konzeptuellen Belegung der freien Variable Q beispielsweise die Deutung in (51k).

Wenn man von so etwas wie einer „,konstruktionsspezifischen Bedeutung $^{\text {c }}$ des Zustandspassivs inklusive etwaiger ereignisbezogener Modifikatoren sprechen möchte, so ist sie genau hier, in der Semantik der Adjektivierungsoperation als Auslöser für den Wortartenwechsel, angelegt - und zwar völlig transparent und kompositional.

Das Prädikat FAHRTAUGLICH in (51k) dient selbstverständlich lediglich als Etikett für sehr viel komplexere Konzeptstrukturen, die im Zuge der konzeptuellen Spezifikation von Q aktiviert werden; siehe hierzu Maienborn (2003b). 


\section{Literatur}

Alexiadou, Artemis/Anagnostopoulou, Elena (2007): Structuring participles. Ms. Univ. Stuttgart/Univ. Kreta.

Anagnostopoulou, Elena (2003): Participles and voice. In: Alexiadou, Artemis/Rathert, Monika/Stechow, Arnim von ( $\mathrm{Hg}$ ): Perfect explorations. (= Interface Explorations 2). Berlin u.a., S. 1-36.

Baker, Mark C. (1988): Incorporation: a theory of grammatical function changing. Chicago u.a.

Barsalou, Lawrence W. (1992): Frames, concepts, and conceptual fields. In: Lehrer, Adrienne/Kittay, Eva Feder $\left(\mathrm{Hg}_{\mathrm{g}}\right)$ : Frames, fields, and contrasts: new essays in semantic and lexical organization. Hillsdale, NJ, S. 21-74.

Bierwisch, Manfred (1988): A puzzling domain in German clause structure. Ms. Berlin.

Embick, David (2004): On the structure of resultative participles in English. In: Linguistic Inquiry 35, S. 355-392.

Frey, Werner (1993): Syntaktische Bedingungen für die Interpretation: Über Bindung, implizite Argumente und Skopus. (= Studia grammatica 35). Berlin.

Frey, Werner (2004): The grammar-pragmatics interface and the German prefield. In: Sprache \& Pragmatik 52, S. 1-39.

Frey, Werner (2006): Contrast and movement to the German prefield. In: Molnár, Valéria/Winkler, Susanne (Hg): Architecture of focus. (= Studies in Generative Grammar 82). Berlin/New York, S. 235-264.

Frey, Werner/Tappe, Hans Thilo (1992): Zur Interpretation der X-bar-Theorie und zur Syntax des Mittelfeldes. Ms. Univ. Stuttgart.

Gese, Helga/Maienborn, Claudia/Stolterfoht, Britta (i.Dr.): Adjectival conversion of unaccusatives in German. In: Journal of Germanic Linguistics.

Gese, Helga/Stolterfoht, Britta/Maienborn, Claudia (2009): Context effects in the formation of adjectival resultatives. In: Winkler, Susanne/Featherston, Sam (Hg): The fruits of empirical linguistics. Bd. 2: Product. (= Studies in Generative Grammar 102). Berlin u.a., S. 231-262.

Gese, Helga/Stolterfoht, Britta (2010): Processing in one step: the integration of event modifiers. Ms. Univ. Tübingen.

Haider, Hubert (1993): Deutsche Syntax - generativ. Vorstudien zur Theorie einer projektiven Grammatik. (= Tübinger Beiträge zur Linguistik 325). Tübingen.

Haider, Hubert (2010): The syntax of German. Cambridge u.a.

Hobbs, Jerry J. et al. (1993): Interpretation as abduction. In: Artificial Intelligence 63, S. 69-142.

Jacobs, Joachim (1993): Integration. In: Reis, Marga ( $\mathrm{Hg}$ ): Wortstellung und Informationsstruktur. (= Linguistische Arbeiten 306). Tübingen, S. 63-116. 
Jacobs, Joachim (1999): Informational autonomy. In: Bosch, Peter/van der Sandt, Rob (Hg.): Focus: linguistic, cognitive, and computational perspectives. Cambridge u.a., S. $56-81$.

Jacobs, Joachim (2004): Zirkumstantien sind Argumente. Ms. Univ. Wuppertal.

Kratzer, Angelika (1994): The event argument and the semantics of voice. Ms. Amherst.

Kratzer, Angelika (2000): Building statives. (= Berkeley Linguistic Society 26). Internet: http://semanticsarchive.net/Archive/GI5MmIOM/kratzer.building.statives.pdf (Stand: 07.12.2010).

Lang, Ewald (1984): The semantics of coordination. (= Studies in Language Companion Series 9). Amsterdam.

Lenz, Barbara (1993): Probleme der Kategorisierung deutscher Partizipien. In: Zeitschrift für Sprachwissenschaft 12, S. 39-76.

Litvinov, Viktor P./Nedjalkov, Vladimir P. (1988): Resultativkonstruktionen im Deutschen. (= Studien zur deutschen Grammatik 34). Tübingen.

Lübbe, Anja (2004): Was heißt Lexikalisierung? Dargestellt am Beispiel deutscher Partizipien. Magisterarbeit. Humboldt-Universität Berlin.

Maienborn, Claudia (1996): Situation und Lokation: Die Bedeutung lokaler Adjunkte von Verbalprojektionen. (= Studien zur deutschen Grammatik 53). Tübingen.

Maienborn, Claudia (2001): On the position and interpretation of locative modifiers. In: Natural Language Semantics 9, 2, S. 191-240.

Maienborn, Claudia (2003a): Die logische Form von Kopula-Sätzen. (= Studia grammatica 56). Berlin.

Maienborn, Claudia (2003b): Event-internal modifiers: semantic underspecification and conceptual interpretation. In: Lang, Ewald/Maienborn, Claudia/Fabricius-Hansen, Cathrine ( $\mathrm{Hg}$ ): Modifying adjuncts. (= Interface Explorations 4). Berlin/New York, S. $475-509$.

Maienborn, Claudia (2005): On the limits of the Davidsonian approach: the case of copula sentences. In: Theoretical Linguistics 31, S. 275-316.

Maienborn, Claudia (2007): Das Zustandspassiv: Grammatische Einordnung - Bildungsbeschränkungen - Interpretationsspielraum. In: Zeitschrift für Germanistische Linguistik 35, S. 83-114.

Maienborn, Claudia (2009): Building event-based ad hoc properties: on the interpretation of adjectival passives. In: Riester, Arndt/Solstad, Torgrim (Hg.): Proceedings of Sinn und Bedeutung 13. Stuttgart, S. 35-49.

Rapp, Irene (1996): Zustand? Passiv? - Überlegungen zum sogenannten Zustandspassiv. In: Zeitschrift für Sprachwissenschaft 15, S. 231-265.

Rapp, Irene (1997): Partizipien und semantische Struktur. Zu passivischen Konstruktionen mit dem 3. Status. (= Studien zur deutschen Grammatik 54). Tübingen. 
Ratcliff, Roger/McKoon, Gail (1978): Priming in item recognition: evidence for the propositional structure of sentences. In: Journal of Verbal Learning and Verbal Behavior 17, S. 403-417.

Schlücker, Barbara (2005): Event-related modifiers in German adjectival passives. In: Maier, Emar et al. (Hg.): Proceedings of Sinn und Bedeutung 9. Nijmegen, S. 417 430.

Solstad, Torgrim (2007): Mehrdeutigkeit und Kontexteinfluss: Die Spezifikation kausaler Relationen am Beispiel von durch. Dissertationsschrift. Univ. Oslo.

Solstad, Torgrim (2010): Some new observations on ,because (of). In: Aloni, Maria et al. $(\mathrm{Hg})$ : Logic, language, and meaning. 17th Amsterdam Colloquium, Amsterdam, The Netherlands, December 16-18, 2009, Revised Selected Papers. (= Lecture Notes in Computer Science 6042). Berlin/Heidelberg, S. 426-445.

Stechow, Arnim von (1998): German Participles II in Distributed Morphology. Ms. Univ. Tübingen.

Steinitz, Renate (1989): $\mathrm{V}^{\mathrm{u}}, \mathrm{I}^{\mathrm{y}}$ und $\mathrm{I}^{z}$ : Überlegungen zum Prädikativ. (= Linguistische Studien des ZISW Reihe A, Arbeitsberichte 194). Berlin (Ost), S. 210-234.

Stolterfoht, Britta/Gese, Helga/Maienborn, Claudia (2010): Word category conversion causes processing costs: evidence from adjectival passives. In: Psychonomic Bulletin \& Review 17, 5, S. 651-656.

Vaagland, Erling M. (1983): Zur Agensangabe im sein-Passiv. In: Askedal, John Ole et al. (Hg.): Festschrift für Laurits Saltveit. Oslo, S. 194-200.

Wunderlich, Dieter (1997): Participle perfect and passive in German. (= Arbeiten des SFB 282 ,Theorie des Lexikons" 99). Düsseldorf.

Zifonun, Gisela/Hoffmann, Ludger/Strecker, Bruno (1997): Grammatik der deutschen Sprache. 3 Bde. (= Schriften des Instituts für Deutsche Sprache 7). Berlin/New York. 